\title{
The Test Ability of an Adaptive Pulse Wave for ADC Testing
}

\author{
Xiaoqin Sheng and Hans G. Kerkhoff \\ CTIT-TDT Group \\ University of Twente \\ Enschede, the Netherlands \\ \{x.sheng, h.g.kerkhoff $\} @$ utwente.nl
}

\begin{abstract}
In the conventional ADC production test method, a high-quality analogue sine wave is applied to the Analogue-toDigital Converter (ADC), which is expensive to generate. Nowadays, an increasing number of ADCs are integrated into a system-on-chip (SoC) platform design, which usually contains a digital embedded processor. In such a platform, a digital pulse wave is obviously less expensive to generate than an accurate analogue sine wave. As a result, the usage of a digital pulse wave has been investigated to test ADCs as the test stimulus. In this paper, the ability of a digital adaptive pulse wave for ADC testing is presented via the measurement results. Instead of the conventional FFT analysis, a timedomain analysis is exploited for post-processing, from which a signature result can be obtained. This signature can distinguish between faulty devices and the fault-free devices. It is also used in the machine-learning-based test method to predict the dynamic specifications of the ADC. The experimental results of a 12-bit $80 \mathrm{M} / \mathrm{s}$ pipelined ADC are shown to evaluate the sensitivity and accuracy of using a pulse wave to test an ADC.
\end{abstract}

Keywords- ADC, test, pulse wave, machine-learning-based, signature, measurement

\section{INTRODUCTION}

In production testing, the multi-site test is a very attractive test method to decrease test time and cost as it can test several chips in parallel [1]. However, for ADC testing, the multi-site test is still limited by the requirement of highquality analogue input stimuli. Nowadays, a large number of ADCs are implemented for multi-media and communication systems as the interface between the analogue world and the digital world. In order to adapt to the fast development of the digital circuitry, the speed and the resolution of the ADCs are increasing rapidly. It also increases the requirements of the quality of the test stimulus, which increases the cost of testing significantly.

Recently, several efforts have been made to decrease the cost of test-signal generation for RF or mixed-signal testing. In [2], a white noise is applied as the test stimulus for ADC testing, which requires low Silicon area overhead for BuiltIn-Self-Test (BIST). With the noise input, the static errors of the Device-Under-Test DUT can be estimated from the spectral analysis. The method has been successfully validated on an 8-bit ADC. In reference [3], the authors exploit a staircase-like exponential waveform as the test stimulus. A pulse-width-modulation (PWM) signal and an off-chip $\mathrm{RC}$ filter are required for generating this input signal. Finally, it is validated that the $3^{\mathrm{rd}}$ harmonic distortion of an ADC up to 20 bits can be tested by using a $3^{\text {rd }}$ order polynomial fitting algorithm. However, the test accuracy is limited by the linearity of the off-chip RC filter. In [4], the machine-learning-based method is applied to test a highspeed ADC. Testing a high-speed ADC requires a highfrequency and high-quality input test signal, which is very expensive to generate in production testing. In order to decrease the expense, the authors exploit two low-frequency signal, a mixer and a band-pass filter to generate the highfrequency signal. However, the quality of the signal is still not sufficient for conventional ADC testing. As a result, only a signature test is carried out with this low-quality signal. Then a mapping function, which is built up via the training data, can map the signature results to the specification space. In this way, the dynamic specifications can be predicted from the signatures. The work in [5] proposes a low-cost test for RF circuits using a relatively low-frequency two-tone signal as the test stimulus. An additional on-chip envelope detector is required for obtaining the wavelet coefficients, which are calculated via the wavelet transforms. Subsequently, the wavelet coefficients are mapped to the specification space by using the machine-learning-based method.

In the past decades, an analogue sine-wave is usually applied as the test stimulus for ADC testing. Looking at the present applications of ADCs, more and more ADCs are integrated with embedded digital processors, which can generate digital signal more easily than an analogue sinewave. As result, in this work we focus on investigating the ability of a pulse wave for testing ADCs by analyzing the measurement results. A time-domain analysis is carried out for post-processing. As result, a signature can be obtained, which can detect the faults in the ADCs. Later, a machinelearning-based test approach is applied to map the signature result to the conventional dynamic specifications of the ADCs. In this way, the dynamic testing of an ADC only requires a simple signature test.

In our previous work [6], [7], we showed the simulation results of using a pulse wave to test ADCs. In this paper, the experimental results are shown and discussed to evaluate the methods as presented in [6] and [7]. The paper is organized as follows. In section 2 , the methods of using a pulse wave 
for ADC testing are presented. The measurement results and analysis of the methods are shown in section 3. The conclusion is presented in the last section.

\section{Methods of Using A Pulse Wave For ADC TESTING}

\section{A. Deviation Comparison by Using Amplitude of the ADC Output}

In our previous work [6], a signature out-of-range percentage (ORP) is introduced, which can distinguish between faulty devices and fault-free devices. An adaptive pulse wave is applied as test stimulus. The basic concept is that of detecting the faults by comparing the output waveforms of the DUTs with the ones of reference devices. A number of golden devices are exploited as the reference devices, which are a collection of the examples of the faultfree devices. When the performance of the ADCs decreases, the values of the ORP become larger. The basic steps for calculating ORP are shown as follows:

Step 1: Apply the pulse wave to the golden devices. For each golden device, an array of the amplitudes $A m$ of the output waveform can be obtained. The element Am(i) represents the amplitude of the ith sampling point. By comparing the output of all the golden devices, the maximum value $A m_{\max }(i)$ and the minimum value $A m_{\min }(i)$ can be obtained for the ith sampling point.

Step 2: Apply the same pulse wave to all the DUTs. An array of the amplitude $A m_{\text {DUT }}$ for each DUT can be obtained as well. For the ith sampling point $A m_{\text {DUT }}(i)$, it is verified if it is within the range $\left[A m_{\min }(i), A m_{\max }(i)\right]$. Otherwise, the absolute value of the amplitude deviation $\triangle A m(i)$ will be calculated.

Step 3: Accumulate the amplitude deviation for all the sampling points to obtain the ORP as:

$$
O R P=\frac{\sum_{i=1}^{N} \Delta A m(i)}{\sum_{i=1}^{N} A m_{\max }(i)-\sum_{i=1}^{N} A m_{\min }(i)}
$$

, where $\mathrm{N}$ is the total number of the sampling points.

\section{B. Machine-learning-based Test Using the Pulse Wave}

The machine-learning-based method is an efficient method for mapping the signature results to the specification results. In this way, the complicated specification test only has to be carried out on the training set. For the DUTs, it can be replaced by the signature test. The basic concept of this method is depicted as in Figure 1. One can see that the important condition to realize the method is that the signature and the specification must be correlated with each other. The better correlation between them, the more accurate estimated specifications can be obtained.

The multivariate adaptive regression splines (MARS) analysis is usually exploited for building the mapping function between the signature and the specifications, as its main function is to predict a dependent variable from a set of independent predictor variables [8].

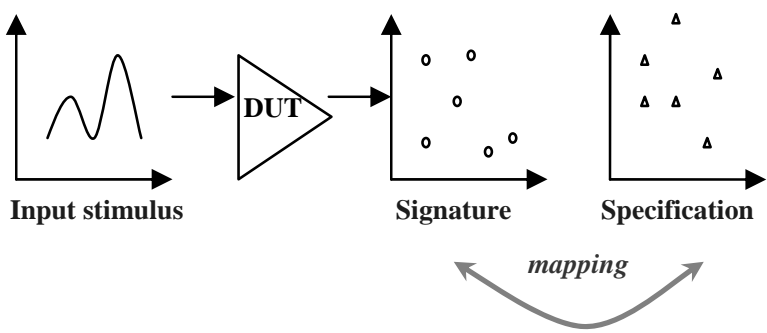

Figure 1. The principle of the machine-learning-based method [7]

As the signature ORP is correlated with the dynamic performance of the ADCs, it is applied for the machinelearning-based method. The machine-learning-based method for testing ADCs can be carried out by the following steps:

Step 1: A number of ADCs are selected as the training set, which should cover all the corner cases for better prediction. Step 2: The conventional specification test is carried out on the training set.

Step 3: The signature test is carried out on the training set.

Step 4: A mapping function can be built between the signature and the specification, which are the input and the output of the function respectively.

Step 5: The signature test is carried out on all the DUTs. The input stimulus should have the same settings as the one in Step 3.

Step 6: The specifications can be calculated by substituting the signature results into the mapping function.

When applying ORP for estimating the dynamic specifications, the selection of the reference devices is different from the one used for distinguishing faulty devices.

- Instead of the golden devices, a number of training devices are exploited as the reference as now the ORP is used for prediction of the specifications.

- Instead of only one ORP of each device to detect the faults, several ORPs are calculated for the prediction of the dynamic specifications. By sorting the values of the training devices dynamic specifications in ascending or descending order, the training devices can be divided into several ranges evenly. As the output waveforms of DUTs are compared with the ones of the training devices from each range, a number of ORPs can be obtained for each device.

\section{Measurement of Using A Pulse Wave to Test ADC}

\section{A. Device-Under-Test}

A 12-bit $80 \mathrm{M} / \mathrm{s}$ pipelined ADC is selected as the target device. It is integrated in the $65 \mathrm{~nm}$ AQUA chip manufactured by NXP, which combines several analogue and mixed-signal blocks in one chip. The pipelined architecture is one of the most popular architectures for 
ADC design. The advantage of this type of ADC is the high resolution, high conversion rate, good dynamic performance and low power consumption. The basic structure of the ADC is shown in Figure 2. One can see that it is composed of 10 stages and the basic structure of each stage is identical as the block denoted by the red dashed line.

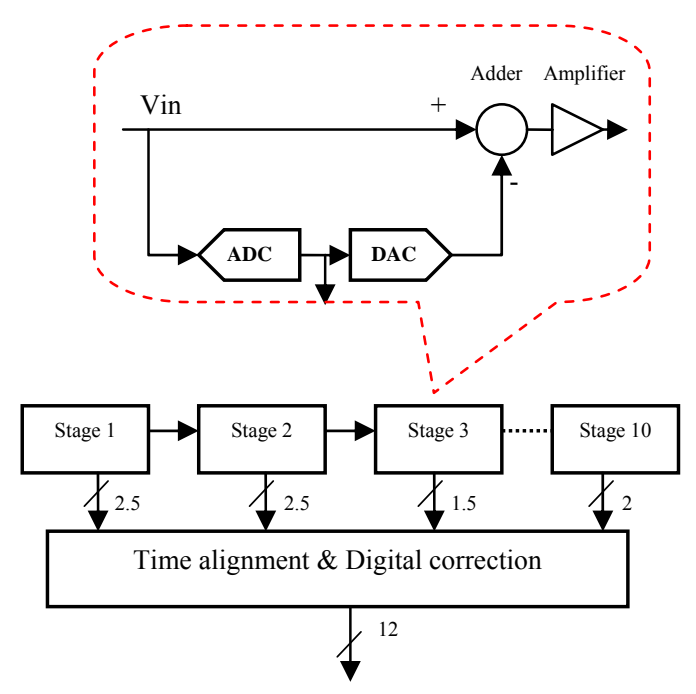

Figure 2. The architecture of the 12-bit pipelined ADC [7]

\section{B. Sensitivity of Detection Faults by Using a Pulse Wave}

\section{1) Emulating Faulty Devices}

In order to emulate faulty devices, we have changed the supply-voltage level of the chip from $0.97 \mathrm{~V}$ to $1.3 \mathrm{~V}$. The normal operation voltage for the ADC is specified from 1.1 $\mathrm{V}$ to $1.3 \mathrm{~V}$. Four important dynamic parameters of the ADC have been considered: total-harmonics-distortion (THD), signal-to-noise-ratio (SNR), signal-to-noise and distortion (SINAD) and spurious free dynamic range (SFDR). They are measured with different levels of the supply voltage. They are all measured with the number of samples of 16384 and a sampling frequency of $25 \mathrm{MHz}$. An analogue sine wave with an input frequency of $1.8 \mathrm{MHz}$ is applied as the test stimulus.

Figure 3 shows the dynamic specifications corresponding to the used supply voltage. One can see that all dynamic parameters follow in the same trend with the supply-voltage level although their values are different. When the supply voltage drops below $1.1 \mathrm{~V}$, the variation of the dynamic parameters is not very obvious at first. If then the supply voltage drops to around $0.98 \mathrm{~V}$, the dynamic performance of the ADC suddenly becomes much worse. In the measurement, the ADC operating at a voltage level below $1.1 \mathrm{~V}$ is taken as the faulty device.

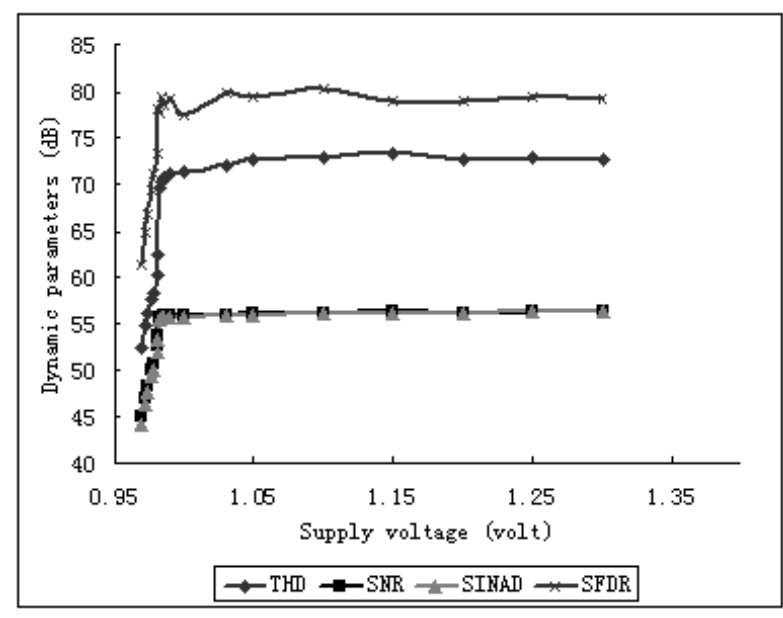

Figure 3. The dynamic specifications vs. supply voltage

2) Fault Detection by Using a Pulse Wave

A signature result ORP is calculated to evaluate the fault if a pulse wave has been used as test stimulus [7]. As explained in section 2.1, when the dynamic performance becomes worse, the value of ORP should increase.

In order to investigate the robustness of the method, five different pulse waves are applied to the device respectively; they have different rise and fall times or number of samples. The parameters of the settings of these pulse waves are listed in Table 1. To emulate the collection of golden devices, the ADC operating at the voltage levels between $1.1 \mathrm{~V}$ and $1.3 \mathrm{~V}$ are used as golden devices. Figure 4 shows the results of ORP versus the supply voltage. Comparing the curves of the ORPs obtained with different pulse wave input stimuli in Figure 4, they are quite close to each other, especially the part where the supply voltage is below $0.98 \mathrm{~V}$. This means that the signature ORP is very robust with regard to the rising and falling edges and the number of samples of the pulse-wave input stimulus.

Comparing Figure 3 with Figure 4, the ORP shows a similar trend as the dynamic specifications with the variation of the supply voltage. One can observe that if the supply voltage drops below $1.1 \mathrm{~V}$, the values of the ORP becomes increasingly larger. However, when it drops around $0.98 \mathrm{~V}$, the slope of the curve suddenly becomes steeper. From section 3.2.1, one knows that the ADC operating at the voltage below $1.1 \mathrm{~V}$ is defined as the faulty device by using the conventional test method. As result, the signature ORP is as sensitive as the conventional dynamic parameters when detecting the faults of the ADC. 


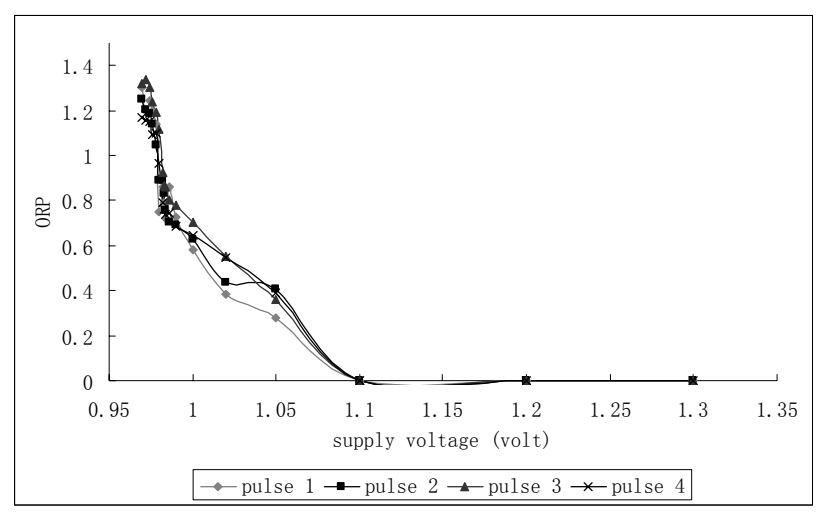

Figure 4. The ORP vs. supply voltage

TABLE I. SETTINGS OF DIFFERENT PULSE WAVE INPUT STIMULUS

\begin{tabular}{|c|l|l|l|l|}
\hline & $\begin{array}{c}\text { Input } \\
\text { frequency } \\
\text { (MHz) }\end{array}$ & $\begin{array}{c}\text { Duty cycle } \\
\mathbf{( \% )}\end{array}$ & $\begin{array}{c}\text { Rise/fall } \\
\text { time } \\
\text { (ns) }\end{array}$ & $\begin{array}{l}\text { Number of } \\
\text { samples }\end{array}$ \\
\hline \hline Pulse1 & 1.8 & 50 & 100 & 4096 \\
\hline Pulse2 & 1.8 & 50 & 100 & 16384 \\
\hline Pulse3 & 1.8 & 50 & 200 & 16384 \\
\hline Pulse4 & 1.8 & 50 & 200 & 32768 \\
\hline
\end{tabular}

\section{Prediction of Dynamic Specifications by Using a Pulse Wave}

As the results show in section 3.2, the signature ORP can distinguish between the faulty devices and the fault-free devices. This is because of the good correlation with the dynamic performance of the ADCs. Based on this conclusion, a machine-learning-based test method is now being introduced, which can map the signature results to the specification results.

In the previous simulation, 2000 training devices are used to build the mapping function and 1500 test devices to validate the method. However, in the real measurement, there are only 109 devices available, which are all manufactured on the same wafer. We divide them in two sets: a training set and a test set. Sixty-three devices are randomly selected as the training devices and the remaining 46 test devices are used to validate the test methods. All DUTs are tested by two main measurements approaches:

1) Conventional testing

The dynamic specifications of the ADCs are measured using a sine wave with an input frequency of $1.8 \mathrm{MHz}$, which is generated by a 16-bit arbitrary waveform generator (AWG). The number of samples is 16384 and the sampling frequency is $25 \mathrm{MHz}$. The THD, SNR, SINAD and SFDR are calculated using the FFT analysis.

\section{2) Signature testing}

In the signature testing approach, five different pulse waves are applied as the test stimulus respectively, in order to investigate the robustness of the method. Their settings are listed in Table 2.
TABLE II. SETTING OF DIFFERENT PULSE INPUT STIMULUS

\begin{tabular}{|c|l|l|l|l|l|}
\hline & $\begin{array}{c}\text { Input } \\
\text { frequency } \\
\text { (MHz) }\end{array}$ & $\begin{array}{c}\text { Duty } \\
\text { cycle } \\
\text { (\%) }\end{array}$ & $\begin{array}{c}\text { Rise/fall } \\
\text { time } \\
\text { (ns) }\end{array}$ & $\begin{array}{c}\text { Linearity } \\
\text { (bits) }\end{array}$ & $\begin{array}{c}\text { Number } \\
\text { of } \\
\text { samples }\end{array}$ \\
\hline \hline Pulse1 & 1.8 & 50 & 100 & 16 & 16384 \\
\hline Pulse2 & 1.8 & 50 & 50 & 16 & 16384 \\
\hline Pulse3 & 1.8 & 50 & 25 & 16 & 16384 \\
\hline Pulse4 & 1.8 & 50 & 100 & 12 & 16384 \\
\hline Pulse5 & 1.8 & 50 & 100 & 7 & 16384 \\
\hline
\end{tabular}

After completing both conventional testing and signature testing, the data from the 64 training devices are used to build the mapping function by using the MARS algorithm as explained in section 2.2. The signature results from the 46 testing devices are used to estimate the dynamic specifications. Then the estimated results are compared with the measured results.

In order to better evaluate the estimated results, the specification testing for each device is repeated ten times. The standard deviation and 3-sigma are calculated as shown in Table 3. The 3-sigma can be interpreted as the maximum error of the conventional specification testing [9]. Here one defines that the estimated results, of which the error is larger than the maximum error, are the outliers.

TABLE III. STANDARD DEVIATION AND 3-SIGMA VALUE OF THE CONVENTIONAL TEST METHOD

\begin{tabular}{|c|l|l|}
\hline & \multicolumn{1}{|c|}{ Std. Deviation } & \multicolumn{1}{c|}{ 3-sigma } \\
\hline \hline THD (dB) & 1 & 3 \\
\hline SNR (dB) & 0.14 & 0.42 \\
\hline SINAD (dB) & 0.14 & 0.42 \\
\hline SFDR (dB) & 1.96 & 5.88 \\
\hline
\end{tabular}

In Figures 5-8, the estimated results obtained by pulse 5 are shown, which has only a 7-bit linearity. In these figures, the $\mathrm{x}$-axis denotes the reference values of the dynamic specifications tested by the specification testing approach. The $y$-axis denotes the values of the dynamic parameters from different data series. The red line denotes the upper and lower limits of the tolerance of the estimated results, which are the reference values minus or plus the 3-sigma value. One can observe that most of the estimated results are in the tolerance band, which means the proposed test method can test the dynamic specifications of the ADC as good as the conventional test method.

The results from the five different pulse wave input stimuli are shown in Table 4. One can notice the difference of the results among them is very small. A pulse-wave input signal with 7-bit linearity (pulse 5) can even obtain similar results as the one with 16-bit linearity (pulse 1). The change of the rising and falling edges do not affect the results in a significant way either when comparing the results obtained from pulse 1, pulse 2 and pulse 3 . It can be concluded that the test method of using a pulse-wave stimulus is very robust for testing the dynamic parameters of the ADCs. 
TABLE IV. MEAN ERROR AND NUMBER OF OUTLIERS WITH DIFFERENT PULSE-WAVE INPUT STIMULUS

\begin{tabular}{|c|c|c|c|c|c|}
\hline & & THD & SNR & SINAD & SFDR \\
\hline \multirow[t]{2}{*}{ Pulse 1} & $\begin{array}{l}\text { Mean error } \\
\text { (dB) }\end{array}$ & 1.61 & 0.11 & 0.25 & 1.36 \\
\hline & $\begin{array}{c}\text { Number of } \\
\text { outlier }\end{array}$ & 2 & 1 & 4 & 2 \\
\hline \multirow[t]{2}{*}{ Pulse 2} & $\begin{array}{l}\text { Mean error } \\
\text { (dB) }\end{array}$ & 1.62 & 0.11 & 0.25 & 1.36 \\
\hline & $\begin{array}{c}\text { Number of } \\
\text { outlier }\end{array}$ & 3 & 2 & 3 & 1 \\
\hline \multirow[t]{2}{*}{ Pulse 3} & $\begin{array}{l}\text { Mean error } \\
\text { (dB) }\end{array}$ & 1.60 & 0.11 & 0.24 & 1.36 \\
\hline & $\begin{array}{c}\text { Number of } \\
\text { outlier }\end{array}$ & 0 & 1 & 2 & 2 \\
\hline \multirow[t]{2}{*}{ Pulse 4} & $\begin{array}{c}\text { Mean error } \\
\text { (dB) }\end{array}$ & 1.62 & 0.12 & 0.26 & 1.36 \\
\hline & $\begin{array}{c}\text { Number of } \\
\text { outlier }\end{array}$ & 1 & 2 & 4 & 1 \\
\hline \multirow[t]{2}{*}{ Pulse 5} & $\begin{array}{l}\text { Mean error } \\
\text { (dB) }\end{array}$ & 1.52 & 0.11 & 0.26 & 1.36 \\
\hline & $\begin{array}{c}\text { Number of } \\
\text { outlier }\end{array}$ & 3 & 1 & 3 & 2 \\
\hline
\end{tabular}

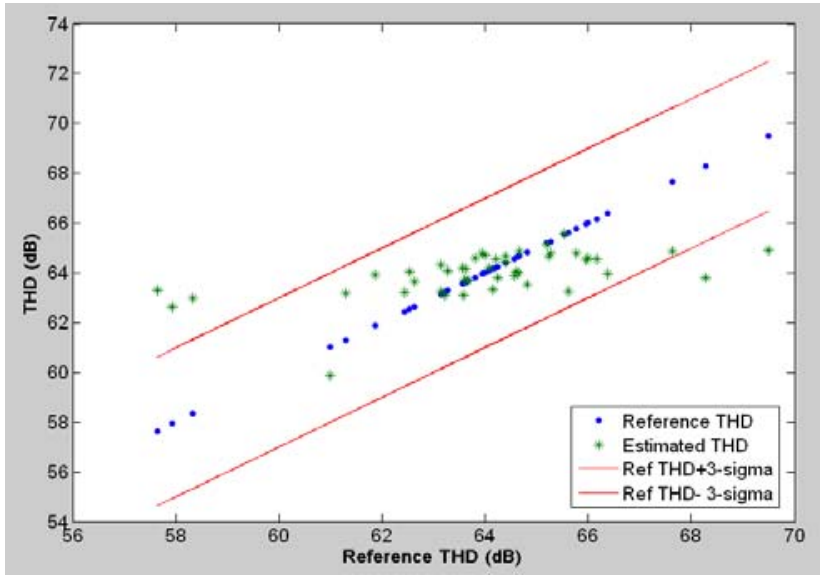

Figure 5. The estimated results of THD with input pulse5

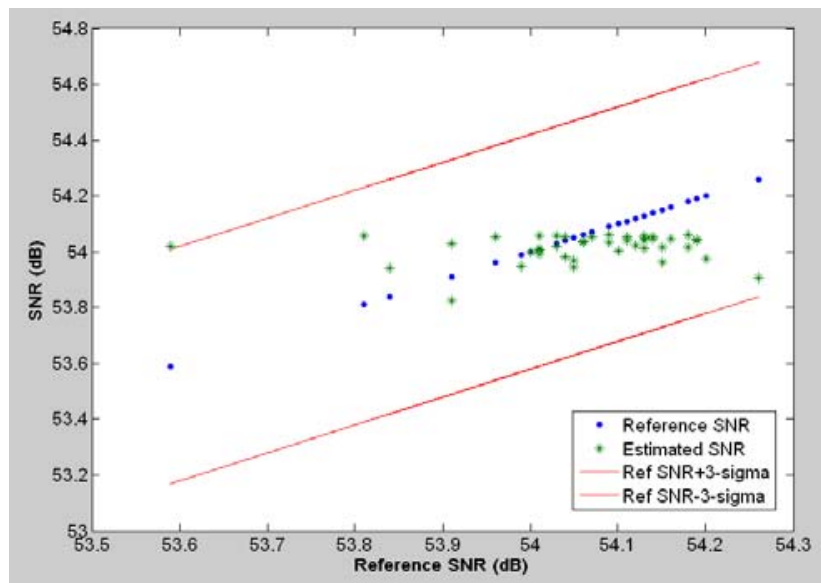

Figure 6. The estimated results of SNR with input pulse 5

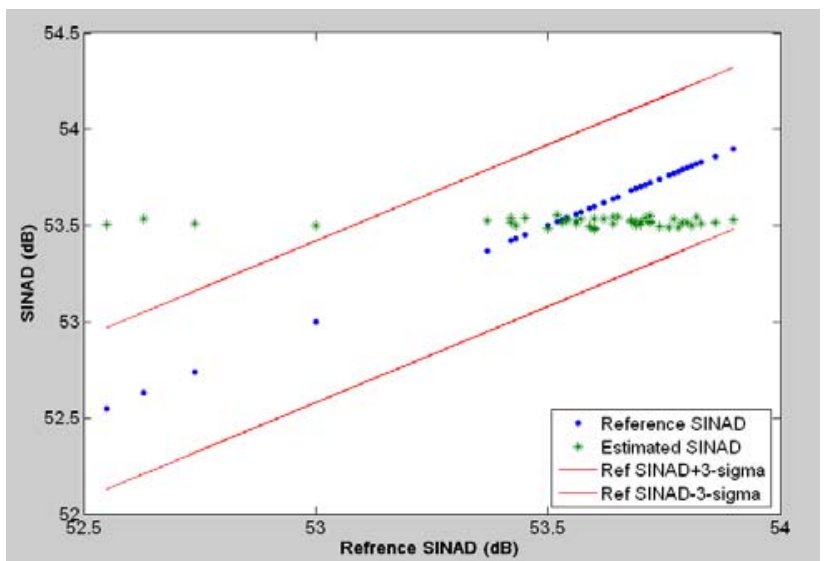

Figure 7. The estimated results of SINAD with input pulse5

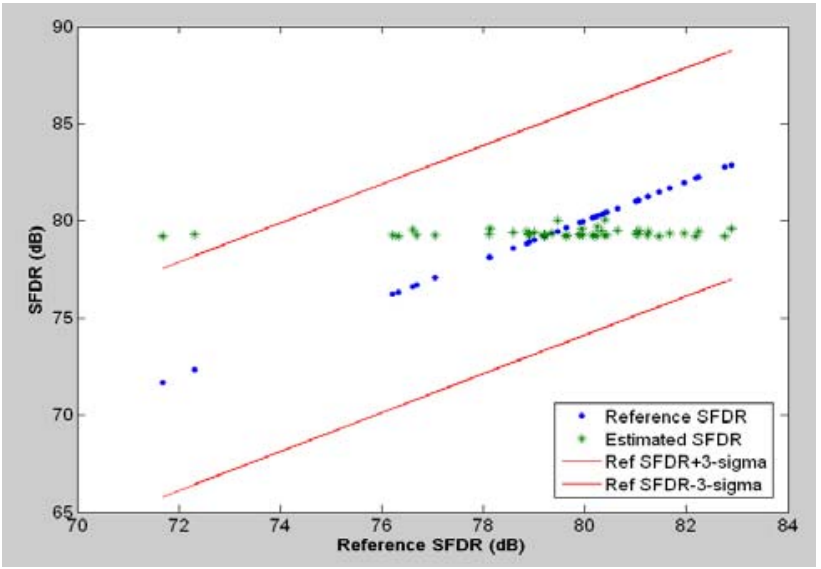

Figure 8. The estimated results of SFDR with input pulse5

\section{CONCLUSION}

In this paper, a pulse-wave input stimulus for ADC testing is discussed and validated via the measurement results. A 12-bit $80 \mathrm{M} / \mathrm{s}$ pipelined ADC is selected as the test vehicle. In our previous work [6] and [7], the test methods of using a pulse wave are only validated by the simulation results. In this work, we focus on the measurement results. The measurement results show that the signature ORP is as sensitive as the dynamic parameters to the faults. As the ORP shows correlation with the dynamic specifications, later on a machine-learning-based method has been investigated by using it as the signature. By using this method, the dynamic specification can be accurately predicted. From the measurement results, one can see that this method is very robust to the slope and linearity of the pulse wave. The dynamic specifications of the 12-bit pipelined ADC can be tested by using a pulse-wave stimulus with 7-bit linearity. However, there are still a few outlier cases, which is quite a common problem in the machine-learning-based test method. As the number of devices is limited now, it is difficult to analyze this issue in this case. In future, this problem can be investigated in more detail when more devices become available. 


\section{REFERENCES}

[1] M. Burns, G. W. Roberts, An Introduction to Mixed-Signal IC Test and Measurement, Oxford University Press, 2000.

[2] M. da Bloria Flores, M. Negreiros, L. Carro, "INL and DNL Estimation based on Noise ADC Test", Instrumentation and Measurement Technology Conference, pp.1350-1353, 2003.

[3] A. Roy, S. Sunter, A. Fudoli, "High Accuracy Stimulus Generation for A/D Converter BIST", International Test Conference, pp. 1031-1039, 2002.

[4] S. Goyal, A. Chatterjee, M. Purtell, "Alternate Test Methodology for High Speed A/D Converter Testing on Low Cost Tester", IEEE Asian Test Symposium, pp. 14-17, 2005.
[5] D. Han, A. Chatterjee, "Robust Built-In Test of RF ICs Using Envelope Detectors", IEEE Asian Test Symposium, pp. 2-7, 2005.

[6] X. Sheng, H. G. Kerkhoff, A. Zjajo, G. Gronthoud, "Algorithms for ADC Multi-Site Test with Digital Input Stimulus", IEEE European Test Symposium, pp. 45-50, 2009.

[7] X. Sheng, V. Kerzérho, H. G. Kerkhoff, "Predicting Dynamic Specifications of ADC with a Low-Quality Digital Input Signal", IEEE European Test Symposium, 2010.

[8] J. H. Friedman "Multivariate Adaptive Regression Splines", The Annals of Statics, vol 19, No.1, 1-141, 1991.

[9] S. Goyal, Efficient Testing of High-performance Data Converters Using Low-Cost Test Instrumentation, $\mathrm{PhD}$ thesis, Georgia Institute of Technology, http://hdl.handle.net/1853/14552, 2007. 